\title{
Light and heavy elements nucleosynthesis in low mass AGB Stars
}

\section{Sergio Cristallo*}

Osservatorio Astronomico di Teramo (INAF)

E-mail: cristallo@oa-teramo.inaf.it

\section{Oscar Straniero}

Osservatorio Astronomico di Teramo (INAF)

E-mail: straniero@oa-teramo.inaf.it

\section{Roberto Gallino}

Universitá degli studi di Torino, Dipartimento di Fisica Generale

E-mail: gallino@ph.unito.it

\section{Luciano Piersanti}

Osservatorio Astronomico di Teramo (INAF)

E-mail: piersanti@oa-teramo.inaf.it

\section{Inma Dominguéz}

Universidad de Granada

E-mail: inma@ugr.es

We present a new set of low mass AGB star models having the same mass $\left(2 \mathrm{M}_{\odot}\right)$ and different metallicities. For each metallicity we follow the evolution from the Pre Main Sequence up to the end of the Thermally Pulsing AGB Phase. By means of an exponential decay of the convective velocities at the inner border of the convective envelope we obtain the formation of a tiny ${ }^{13} \mathrm{C}$ pocket after Third Dredge Up episodes, whose extension in mass decreases along the AGB path. Detailed pulse by pulse surface enrichments and final yields at different metallicities, computed by using a full nuclear network coupled to the FRANEC stellar evolutionary code, are presented and discussed. We follow the production of both light and heavy elements describing nuclear chains responsible for their production and show new results for the synthesis of radioactive isotopes such as ${ }^{26} \mathrm{Al}$ and ${ }^{60} \mathrm{Fe}$.

International Symposium on Nuclear Astrophysics - Nuclei in the Cosmos - IX

June 25-30 2006

CERN, Geneva, Switzerland

\footnotetext{
${ }^{*}$ Speaker.
} 


\section{Introduction}

The abundances in the Solar System are due to the mixing of material ejected from stars that polluted the interstellar matter before the formation of the Sun. Isotopes heavier than iron $(A \geq 56)$ are created by means of neutron capture processes: by the $\mathrm{r}$ process, which requires very high neutron densities $\left(n_{n} \gtrsim 10^{20} \mathrm{~cm}^{-3}\right)$ and is believed to occur during the explosive phases at the end of the evolution of massive stars, and by the s process, active during the Thermally Pulsing Asymptotic Giant Branch (TP-AGB) phase of low mass stars $\left(1.5 \lesssim \mathrm{M} / \mathrm{M}_{\odot}<4\right)$ [1]. In this phase, the stellar structure consists of a partially degenerate carbon-oxygen core, a He shell separated from a $\mathrm{H}$ shell by a small He-rich region (He-intershell), and a convective envelope. The energy required to balance the surface irradiation is mainly provided by the $\mathrm{H}$ burning shell, located just below the inner border of the convective envelope. This situation is recurrently interrupted by a thermonuclear runaway (Thermal Pulse, TP) in the He-shell; as a consequence of a TP, the He-intershell becomes unstable to convection for a short period, the external layers expand and the $\mathrm{H}$ shell burning temporarily dies down. After this episode, the convective envelope can penetrate in the C-rich He-intershell (Third Dredge Up episode, TDU), carrying out to the surface the freshly synthesized ${ }^{12} \mathrm{C}$ and s process elements. In AGB stars, the main neutron source is the ${ }^{13} \mathrm{C}(\alpha, n){ }^{16} \mathrm{O}$ reaction. The burning of the ${ }^{13} \mathrm{C}$ left in the $\mathrm{H}$-shell ashes produces a negligible neutron exposure: this is due to the fact that, in the material processed by the $\mathrm{CNO}$ burning, the ${ }^{14} \mathrm{~N}$ (a strong neutron poison) is, in any case, two orders of magnitude more abundant than the ${ }^{13} \mathrm{C}$. Then, an alternative source of ${ }^{13} \mathrm{C}$ is needed, in a zone where ${ }^{14} \mathrm{~N}$ is depleted: the so-called ${ }^{13} \mathrm{C}$ pocket. This pocket can form if, at the moment of the maximum penetration of the convective envelope during TDU episodes, a small amount of protons are injected into the radiative He-intershell, where the ${ }^{14} \mathrm{~N}$ has been previoulsy converted to ${ }^{22} \mathrm{Ne}$ by means of $\alpha$ captures. Later on, when this region heats up again, protons are captured by the abundant ${ }^{12} \mathrm{C}$ via the sequence ${ }^{12} \mathrm{C}(\mathrm{p}, \gamma){ }^{13} \mathrm{~N}\left(\beta^{+} v\right){ }^{13} \mathrm{C}$ and subsequently, when the temperature reaches about $10^{8} \mathrm{~K}$, the ${ }^{13} \mathrm{C}(\alpha, \mathrm{n}){ }^{16} \mathrm{O}$ reaction is efficiently activated in the pocket, producing a large amount of neutrons which are captured by the iron seeds. The problem of mixing protons into a thin layer of the He-intershell, to produce the so-called ${ }^{13} \mathrm{C}$ pocket, presents a challenge in stellar modelling: we attack it by introducing an exponentially decaying profile of the convective velocities at the inner border of the convective envelope. In Sec. 2 we report computations of a star with $M=2 M_{\odot}$ at different chemical compositions: these models have been obtained by including into the FRANEC stellar evolutionary code a full nuclear network (from $\mathrm{H}$ up to Bi, at the termination point of the s process path), upgraded with the most recent experimental and theoretical cross sections. The predicted modifications of the surface compositions occurring during the AGB evolution are shown in Section 3. Moreover, we compare our theoretical distributions with Galactic s process enriched stars. Finally, in Section 4 we report our conclusions.

\section{The models}

We compute two models with the same initial mass $\left(M=2 M_{\odot}\right.$, assumed as representative of low mass AGB stars) with different metallicities $\left(Z=1.5 \times 10^{-2} \equiv Z_{\odot}\right.$ and $\left.Z=1 \times 10^{-4}\right)$, representative of galactic disk and halo stars, respectively. For a detailed description of the physical evolution of these models we refer to $[2,3]$ : here we concentrate on the chemical evolution. With respect 
to previous versions of the stellar code (see [3] and references therein) a different treatment of the opacity coefficients has been introduced and a new mass loss rate has been adopted. The opacity changes, caused by the variation of the internal chemical composition due to nuclear burning, convective mixing and microscopic diffusion, are now taken into account by linearly interpolating between tables with different $Z$ and $Y$. This procedure guarantees an accurate determination of the surface abundance variations, provided that the relative distribution of the heavy $(Z \geq 6)$ elements remains almost unchanged. However, AGB envelopes become largely enriched in carbon after TDU episodes (mainly at low metallicities), which has strong consequences for the determination of the opacity coefficients (we refer to Section 3 for the analysis of this problem). Concerning the second point, we derive a theoretical mass loss rate as a function of the pulsation period, by analysing a sample of Galactic C-rich and O-rich AGB stars (see $[4,2]$ and references therein):

- for $\log P<2.5$

$$
\dot{M}=1.34 \times 10^{-5} \cdot \eta \cdot \frac{L^{\frac{3}{2}}}{M \cdot T_{\mathrm{eff}}^{2}}(\eta=0.4) ;
$$

- for $2.5 \leq \log P \leq 3.1 \quad \dot{M}=\max \left\{\begin{array}{l}1.34 \times 10^{-5} \cdot \eta \cdot \frac{L^{\frac{3}{2}}}{M \cdot T_{\text {eff }}^{2}}(\eta=0.4) \\ 10^{\left[-101.6+63.26 \cdot \log P-10.282 \cdot(\log P)^{2}\right]}\end{array}\right.$;

- for $\log P>3.1 \quad \dot{M}=5 \times 10^{-5}$.

The introduction of a physical algorithm for the treatment of the convective/radiative interfaces (see $[5,3])$ allows the partial diffusion of protons in the top layers of the He shell, giving naturally rise to the subsequent formation of a tiny ${ }^{13} \mathrm{C}$-rich layer: the so-called ${ }^{13} \mathrm{C}$ pocket. This pocket partially overlaps with an outer ${ }^{14} \mathrm{~N}$ pocket, followed by a further minor ${ }^{23} \mathrm{Na}$ pocket, which originates from proton captures on the abundant ${ }^{22} \mathrm{Ne}$ (see [6]). The extension in mass of the ${ }^{13} \mathrm{C}$-pocket decreases along the AGB path. The ${ }^{13} \mathrm{C}$ burns completely in radiative conditions during the interpulse phase, releasing neutrons via the ${ }^{13} \mathrm{C}(\alpha, \mathrm{n}){ }^{16} \mathrm{O}$ reaction, with the exception of the first pocket of the solar metallicity model (see next Section).

\section{Nucleosynthesis results}

In Table 1 we report, in the usual spectroscopic notation ${ }^{1}$, the final distributions corresponding to the solar metallicity case (column 2) and to the low metallicity case (columns 3 and 4): the label st and test refer to models computed by interpolating the opacity coefficients with the metallicity or by using opacity tables at $Z=1 \times 10^{-4}$ for the entire AGB evolution, respectively. This has been done in order to explore the sensitivity of the physical and chemical evolution of the model with respect to the adopted opacity treatment. In Table 2 we report the total cumulative yields of the three models; an extended electronic version of Tables 1 and 2 is available on the $\mathrm{web}^{2}$.

Concerning the solar metallicity model, light elements are mildly enhanced: while $\mathrm{C}$ is the main product of the partial $3 \alpha$-burning during the TPs and is mixed with the envelope by TDU episodes, the surface nitrogen enrichment is totally due to the first dredge up. Fluorine is synthesized in the convective shell generated by the TP by means of the ${ }^{15} \mathrm{~N}(\alpha, \gamma){ }^{19} \mathrm{~F}$ reaction, while the

\footnotetext{
${ }^{1}[\mathrm{El} / \mathrm{Fe}]=\log (N(\mathrm{El}) / N(\mathrm{Fe}))-\log (N(\mathrm{El}) / N(\mathrm{Fe})) \odot$. The label El stands for the generic element.

${ }^{2} \mathrm{http} / / /$ www.oa-teramo.inaf.it/osservatorio/personale/cristallo/data_online.html .
} 


\begin{tabular}{lccc}
\hline & $Z=Z_{\odot}$ & $Z=1 \times 10^{-4}{ }_{s t}$ & $Z=1 \times 10^{-4}{ }_{\text {test }}$ \\
\hline \hline$[\mathrm{C} / \mathrm{Fe}]$ & $5.6 \mathrm{E}-01$ & $2.6 \mathrm{E}+00$ & $3.3 \mathrm{E}+00$ \\
{$[\mathrm{~N} / \mathrm{Fe}]$} & $3.0 \mathrm{E}-01$ & $6.4 \mathrm{E}-01$ & $1.5 \mathrm{E}+00$ \\
{$[\mathrm{O} / \mathrm{Fe}]$} & $2.1 \mathrm{E}-03$ & $7.4 \mathrm{E}-01$ & $1.3 \mathrm{E}+00$ \\
{$[\mathrm{~F} / \mathrm{Fe}]$} & $4.9 \mathrm{E}-01$ & $2.4 \mathrm{E}+00$ & $3.7 \mathrm{E}+00$ \\
{$[\mathrm{Na} / \mathrm{Fe}]$} & $1.7 \mathrm{E}-01$ & $1.0 \mathrm{E}+00$ & $2.8 \mathrm{E}+00$ \\
{$[\mathrm{ls} / \mathrm{Fe}]$} & $1.1 \mathrm{E}+00$ & $6.4 \mathrm{E}-01$ & $1.7 \mathrm{E}+00$ \\
{$[\mathrm{hs} / \mathrm{Fe}]$} & $8.1 \mathrm{E}-01$ & $1.1 \mathrm{E}+00$ & $2.3 \mathrm{E}+00$ \\
{$[\mathrm{~Pb} / \mathrm{Fe}]$} & $5.3 \mathrm{E}-01$ & $2.6 \mathrm{E}+00$ & $3.1 \mathrm{E}+00$ \\
\hline \hline
\end{tabular}

Table 1: Final surface overabundances (in the usual spectroscopic notation) for the $Z=Z \odot$ model (column 2) and for the $Z=1 \times 10^{-4}$ model (column 3 and 4 ). The $s t$ label refers to a model computed by interpolating in metallicity the opacity coefficients, while the test one to a model where the opacity coefficients are calculated with a fixed (the initial one) metallicity.

low $\mathrm{Na}$ enhancement is mainly due to proton capture on ${ }^{22} \mathrm{Ne}$, active during the formation of the ${ }^{23} \mathrm{Na}$ pocket (see previous Section). All heavy elements (from $\mathrm{Sr}$ to $\mathrm{Pb}$ ) are enhanced: we find that the abundance of the so-called ls elements ( $\mathrm{Sr}, \mathrm{Y}$ and $\mathrm{Zr}$ ) is comparable with the one of the hs elements (Ba, $\mathrm{La}, \mathrm{Ce}, \mathrm{Pr}, \mathrm{Nd})$. Lead is underproduced with respect to barium, as expected for AGB stars of this metallicity. We compare our surface enrichment distribution with the one obtained with a post-process technique [7], where a constant pulse by pulse ${ }^{13} \mathrm{C}$ pocket is artificially introduced. This calculation has been performed on a $M=2 M_{\odot}$ model with $Z=2 \times 10^{-2}$ [8], which is characterized by a Reimers' mass-loss rate with $\eta=0.5$. By varying the efficiency of the parameterized ${ }^{13} \mathrm{C}$ pocket, we find that our model is approximately equivalent to the ST $\times 1.3$ case of [7]: the average enhancement of s process elements is roughly the same, even if the distributions result from completely different evolutions along the AGB phase. We in fact obtain 11 pulses followed by TDU, while the post-process calculation runs over a model characterized by 22 pulses followed by TDU [8]. However, we recall that the introduction of the velocity profile algorithm makes our TDU more efficient with respect to previous calculations: this feature counterbalances the decreased number of TDU episodes, which is mainly determined by the adopted mass-loss rate. In order to evaluate

\begin{tabular}{lccc}
\hline & $Z=Z_{\odot}$ & $Z=1 \times 10^{-4}{ }_{s t}$ & $Z=1 \times 10^{-4}{ }_{\text {test }}$ \\
\hline \hline${ }^{12} \mathrm{C}$ & $6.0 \mathrm{E}-03$ & $7.9 \mathrm{E}-03$ & $3.1 \mathrm{E}-02$ \\
${ }^{14} \mathrm{~N}$ & $1.4 \mathrm{E}-03$ & $2.6 \mathrm{E}-05$ & $1.2 \mathrm{E}-04$ \\
${ }^{16} \mathrm{O}$ & $-1.0 \mathrm{E}-04$ & $2.1 \mathrm{E}-04$ & $6.6 \mathrm{E}-04$ \\
${ }^{19} \mathrm{~F}$ & $9.3 \mathrm{E}-07$ & $7.8 \mathrm{E}-07$ & $1.2 \mathrm{E}-05$ \\
${ }^{23} \mathrm{Na}$ & $2.1 \mathrm{E}-05$ & $2.7 \mathrm{E}-06$ & $1.2 \mathrm{E}-04$ \\
${ }^{26} \mathrm{Al}$ & $3.9 \mathrm{E}-07$ & $1.4 \mathrm{E}-08$ & $2.6 \mathrm{E}-07$ \\
${ }^{60} \mathrm{Fe}$ & $1.1 \mathrm{E}-07$ & $6.7 \mathrm{E}-09$ & $2.2 \mathrm{E}-08$ \\
\hline \hline
\end{tabular}

Table 2: As in Table 1, but referring to cumulative yields. All tabulated values are in $M_{\odot}$. 
the efficiency of the adopted velocity profile algorithm, we compare our surface [hs/ls] value with a sample of solar metallicity galactic $\mathrm{C}(\mathrm{N})$ Giants [9] (left panel of Fig. 1): since their mean C/O surface ratios are about 1 , we report our envelope composition for $\mathrm{C} / \mathrm{O}=1$, which is attained after the $6^{\text {th }}$ pulse with TDU. The value we find lies in the range spanned by observational data.

Other interesting by-products of AGB nucleosynthesis are the short-lived isotopes, whose role is of primary importance in the determination of the astrophysical source which polluted the Early Solar System [10]: ${ }^{26} \mathrm{Al}$ and ${ }^{60} \mathrm{Fe}$ belong to this class. The surface of our $Z=Z_{\odot}$ model is enriched of freshly synthesized ${ }^{26} \mathrm{Al}$ as a consequence of TDU episodes $\left({ }^{26} \mathrm{Al}\right.$ is efficiently produced by proton captures on ${ }^{25} \mathrm{Mg}$ in the H-burning shell). In fact, the temperatures attained at the bottom of the convective TPs are not high enough to efficiently activate the ${ }^{22} \mathrm{Ne}(\alpha, \mathrm{n})^{25} \mathrm{Mg}$ reaction and, consequently, the ${ }^{26} \mathrm{Al}$ is only weakly destroyed by the ${ }^{26} \mathrm{Al}(\mathrm{n}, \mathrm{p})^{26} \mathrm{Mg}$ reaction. We therefore find a substantial surface enhancement of ${ }^{26} \mathrm{Al}$ (the final surface aluminum isotopic ratio is $\left.\left.\left.X\left({ }^{26} \mathrm{Al}\right)\right) / X\left({ }^{27} \mathrm{Al}\right)\right)=5.3 \times 10^{-3}\right)$. The ${ }^{60} \mathrm{Fe}$ production is instead mainly due to a single convective ${ }^{13} \mathrm{C}$ burning episode, when the first ${ }^{13} \mathrm{C}$ pocket is engulfed by the convective shell generated by the following TP (see [6]). In this case the ${ }^{13} \mathrm{C}$ burns at higher temperatures $\left(T_{8} \sim 1.6\right)$, producing a larger neutron density with respect to a radiative ${ }^{13} \mathrm{C}$ burning. This fact has minor consequences on the physical and chemical evolution of the star, except for the nucleosynthesis of neutron-rich isotopes; accordingly, our final ${ }^{60} \mathrm{Fe}$ surface abundance is increased $\left(X\left({ }^{60} \mathrm{Fe}\right) / X\left({ }^{56} \mathrm{Fe}\right)=6.2 \times 10^{-5}\right)$ with respect models where a standard ${ }^{13} \mathrm{C}$ burning is taken into account (see [10]). Even if we obtain more ${ }^{60} \mathrm{Fe}$, we can't claim that an AGB star has been the unique polluter of the Early Solar System (ESS), because of a too large palladium production (see discussion in [6]): in order to solve this problem, [10] postulated that the ESS was polluted from an AGB star with $M=3 M$. and $Z=\frac{1}{3} Z_{\odot}$, with $\mathrm{NO}{ }^{13} \mathrm{C}$ pockets. Theories claiming that a Type II Supernova was the unique polluter of the ESS are instead dealing with a too large ${ }^{53} \mathrm{Mn}$ production (see [10]). Useful hints on this debated argument could come from the work of Korschinek et al. (these proceedings), who detected ${ }^{60} \mathrm{Fe}$ in a deep-sea ferromanganese crust, compatible with the deposition of ejecta from a nearby SN.

Concerning the low metallicity cases, the data in Tables 1 and 2 clearly indicate how the opacity treatment affects the model evolution (for a detailed analysis see Section 8 of [2]). In particular, the pulse number increases from 9 (st case) to 49 (test case), which has strong consequences on the chemical surface enrichment: it appears largely enhanced in the test case as compared to the st case. Note that the choice of a lower mass loss rate should increase the number of TPs as well, allowing the model to reach larger surface overabundances. A useful guideline in identifying the best model comes from spectroscopic measurements of low metallicity halo stars (see Table 1 in [11]): the majority of these stars show larger $[\mathrm{hs} / \mathrm{Fe}]$ values (up to with $\sim 2.3$ ) with respect to the theoretical expectation found in the st case. The observed level is attained by our test case: at the moment, we therefore assume the test case as our best model at low metallicity. As expected at these metallicities, we find a strong carbon enhancement $([\mathrm{C} / \mathrm{Fe}]=3.3)$, consequence of the TDU episodes, and a large lead overabundance $([\mathrm{Pb} / \mathrm{Fe}]=3.1)$. While the ${ }^{13} \mathrm{C}(\alpha, \mathrm{n}){ }^{16} \mathrm{O}$ is of primary origin, the iron seeds scale with metallicity, resulting in a larger number of neutrons per iron seed. At low metallicities, most of the seeds are consequently converted to ${ }^{208} \mathrm{~Pb}$, at the termination point of the s process [12]. The large carbon and fluorine surface enrichments are the direct consequences of the increased number of TDU episodes, while the final nitrogen increase is instead due to the 

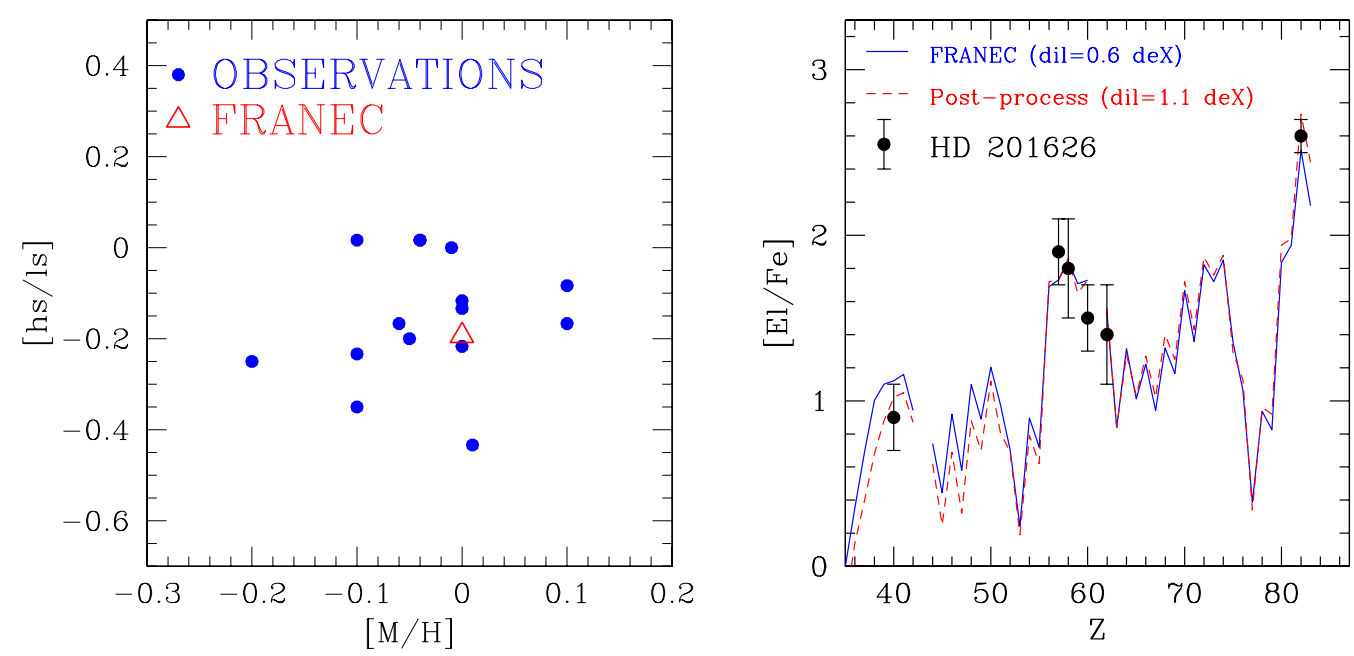

Figure 1: Left Panel: observed [hs/ls] ratio of a sample of galactic C(N) Giants [9] (blue circles), compared with our theoretical prediction (red triangle); Right Panel: theoretical expectations (blue solid line: FRANEC [this work]; red dotted line: post-process calculation [12]) compared with spectroscopic data of and HD 201626 [13].

mixing with the envelope, during TDU episodes, of the $\mathrm{H}$ shell and the underlying layers (which are enriched in ${ }^{14} \mathrm{~N}$ ). Sodium production occurs at the epoch of the formation of the ${ }^{13} \mathrm{C}$ pocket, when proton captures on ${ }^{22} \mathrm{Ne}$ give rise to a tiny ${ }^{23} \mathrm{Na}$ pocket, and during the convective TPs, where the abundant primary ${ }^{22} \mathrm{Ne}$ in the He-intershell captures a fraction of the neutrons released by the activation of the ${ }^{22} \mathrm{Ne}(\alpha, \mathrm{n})^{25} \mathrm{Mg}$ reaction (see also the contribution from Gallino et al., these proceedings). In the right panel of Fig. 1 we compare our theoretical expectation (solid blue curve) with spectroscopic measurements of the lead star HD 201626 [13]; in the same Figure we also plot the curve obtained with the post-process calculation [12] (red dotted line), relative to a ST/3 case of a $M=1.5 M_{\odot}$ star with $Z=2 \times 10^{-4}$. Note that a dilution factor is required for both theoretical curves: this implies that this star is an extrinsic AGB star, in agreement with the work of [14], who determined the orbital elements of the system to which this star is belonging.

\section{Conclusions}

The self-consistent formation of a ${ }^{13} \mathrm{C}$ pocket allows us to evaluate the s process surface enrichment of low mass AGB stars and to compare it with the extant spectroscopic measurements. We found a good agreement with observations at both metallicities, even if a single model cannot reproduce the abundances spread, observed at low metallicities [12]. The resulting final surface abundances seem to suggest that the model computed with opacity tables at fixed metallicity (test case) is more efficient in reproducing spectroscopic data at low metallicities, while the model where the metal enrichment of the envelope is taken into account ( $s t$ case) seems to overstimate the atomic contribution to the opacity. This discrepancy turns our attention to the problem of the molecular contribution to opacity, which has been demonstrated to be dominant in the external layers of the 
cool AGB envelopes when the $\mathrm{C} / \mathrm{O}$ ratio is bigger than 1 (see [15]): we deserve this analysis to future works.

Moreover, it has to be stressed that the low number of thermal pulses in the st case is a consequence not only of the opacity coefficients treatment, but it depends also on the adopted mass-loss rate. The large scatter in the observational data cannot obviously be reproduced using a single theoretical fitting curve: it is therefore necessary to investigate the effect of reducing the adopted mass-loss on the number of termal pulses by means a new set of models and to evaluate the consequences on the following chemical evolution.

\section{References}

[1] Busso, M., Gallino, R., Wasserburg, G. J.: 1999, ARA\&A 37, 239.

[2] Cristallo, S.: 2006, PASP, 118, 1363.

[3] Chieffi, A., Domínguez, I., Limongi, M., Straniero, O.: 2001, ApJ 554, 1159.

[4] Straniero, O., Gallino, R., Cristallo, S.: 2006, Nucl. Phys. A 777, 311.

[5] Cristallo, S., and 6 coauthors: 2001, Nucl. Phys. A, 688, 217.

[6] Cristallo, S., Gallino. R., Straniero, O., Piersanti, L., Domínguez, I.: 2006, Mem. SAIt 77, 774.

[7] Gallino, R., and 7 coauthors.: 1998, ApJ 497, 388.

[8] Straniero, O., Domínguez, I., Cristallo, S., Gallino, R.: 2003, PASA 20, 389.

[9] Abia, C., and 8 coauthors: 2002, ApJ 579, 817.

[10] Wasserburg, J.J., Busso, M., Gallino, R., Nollett, K.M.: 2006, Nucl. Phys. A 777, 5.

[11] Bisterzo, S., and 5 coauthors: 2006, OMEG05 Symposium, in press.

[12] Gallino, R., Arnone, E., Pignatari, M., Straniero, O.: 2005, Mem. SAIt 75, 700.

[13] Van Eck, S., Goriely, S., Jorissen, A., Plez, B.: 2003, A\&A 404, 291.

[14] McClure, R.D., \& Woodsworth, A.W.: 1990, ApJ 352, 709.

[15] Marigo, P.: 2002, A\&A 387, 507. 